\title{
Failure After Failure. What Next in AD Drug Development?
}

\author{
P.S. Aisen \\ Alzheimer's Therapeutic Research Institute (ATRI), Keck School of Medicine, University of Southern California, San Diego, CA, USA

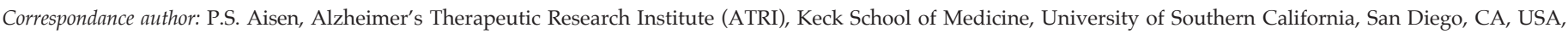 \\ paisen@usc.edu
}

$\mathbf{T}$ The Phase 3 aducanumab program in early $\mathrm{AD}$ has been stopped based on a futility efficacy analysis. This huge disappointment follows exciting preliminary studies that had raised expectations (1). Now this frustrating result joins the long series of negative and interrupted trials of potential diseasemodifying agents, going back to tarenflurbil (2), tramiprosate (3), and bapineuzumab (4), among many others. Is the amyloid hypothesis wrong? Where do we go from here?

Until we have successful development of an antiamyloid treatment that yields clinically meaningful benefits, the hypothesis remains unproven. Some have long decried the field's focus on amyloid as the most promising target and amyloid naysayers are now growing in number. David Knopman concluded his recent editorial on beta secretase failures, "To be blunt, $A \beta$ lowering seems to be an ineffective approach, and it is time to focus on other targets to move therapeutics for Alzheimer's disease forward" (5).

Certainly we must pursue other strategies. Immunotherapeutic attacks on tau pathology, apolipoprotein E-based therapeutics, anti-microbial agents, anti-inflammatories and non-pharmacological approaches are among many strategies worthy of investigation. We must select the most compelling ideas and test them in well-powered clinical trials. Combining therapies may improve the likelihood of success. The enormous need is inarguable. Failures must not lead to capitulation.

But it is not time to abandon or even lose our focus on anti-amyloid interventions. Even as many specific approaches can now be discarded, the rationale remains strong. Anti-amyloid immunotherapy seems unlikely to yield substantial disease slowing at the dementia stage (6). Strong inhibition of gamma-secretase $(7,8)$ or betasecretase $(9,10)$ may not be helpful in symptomatic $A D$, and carries risk of cognitive worsening. But convincing evidence, particularly from genetic studies in autosomal dominant and Dpwn syndrome-associated AD as well as sporadic AD, points to amyloid as causative (11). Amyloid drives disease onset, but the extent of amyloid in brain does not correlate with disease progression, and it may be reasonable to conclude that the advancing neurodegeneration and accumulating comorbidities associated with symptomatic AD may limit the therapeutic potential of targeting amyloid at later stages. But the approach with the strongest rationale, targeting amyloid before any symptoms occur, has not yet been tested. The A4 trial (12), testing an antimonomeric amyloid antibody, is fully enrolled with 1169 randomized participants, but will not be completed until late in 2022. Other anti-amyloid immunotherapy trials in pre-symptomatic stages will be launched in the coming months. And relatively low dose beta-secretase inhibition therapy, to inhibit the enzyme only $50 \%$ or less, remains a plausible approach to very early intervention against amyloid accumulation, or primary prevention of the disease.

Despite multiple failures, it is not time to abandon the hypothesis that has the strongest rationale. Success may still be within reach.

Conflict of interest: Dr. Aisen has grants from Lilly, Janssen, NIH, FNIH and the Alzheimer's Association, and has received consulting fees from Biogen, Merck, Roche, Proclara, Eisai, Lundbeck and ImmunoBrain Checkpoint.

\section{References}

1. Sevigny J, Chiao P, Bussiere T, et al. The antibody aducanumab reduces Abeta plaques in Alzheimer's disease. Nature 2016;537:50-6.

2. Green RC, Schneider LS, Amato DA, et al. Effect of tarenflurbil on cognitive decline and activities of daily living in patients with mild Alzheimer disease: a randomized controlled trial. JAMA 2009;302:2557-64.

3. Aisen PS, Gauthier S, Ferris SH, et al. Tramiprosate in mild-to-moderate Alzheimer's disease - a randomized, double-blind, placebo-controlled, multicentre study (the Alphase Study). Arch Med Sci 2011;7:102-11.

4. Salloway S, Sperling R, Fox NC, et al. Two phase 3 trials of bapineuzumab in mild-to-moderate Alzheimer's disease. N Engl J Med 2014;370:322-33.

5. Knopman DS. Lowering of Amyloid-Beta by $\beta$-Secretase Inhibitors - Some Informative Failures. NEJM 2019;380.

6. Salloway S, Sperling R, Brashear HR. Phase 3 trials of solanezumab and bapineuzumab for Alzheimer's disease. N Engl J Med 2014;370:1460.

7. Doody RS, Raman R, Farlow M, et al. A phase 3 trial of semagacestat for treatment of Alzheimer's disease. N Engl J Med 2013;369:341-50.

8. Coric V, Salloway S, van Dyck CH, et al. Targeting Prodromal Alzheimer Disease With Avagacestat: A Randomized Clinical Trial. JAMA Neurol 2015;72:1324-33.

9. Egan MF, Kost J, Tariot PN, et al. Randomized Trial of Verubecestat for Mildto-Moderate Alzheimer's Disease. N Engl J Med 2018;378:1691-703.

10. Egan MF, Kost J, Voss T, et al. Randomized Trial of Verubecestat for Prodromal Alzheimer's Disease. NEJM 2019;380.

11. Selkoe DJ, Hardy J. The amyloid hypothesis of Alzheimer's disease at 25 years. EMBO Mol Med 2016;8:595-608.

12. Sperling RA, Rentz DM, Johnson KA, et al. The A4 study: stopping AD before symptoms begin? Sci Transl Med 2014;6:228fs13. 